\title{
Event-Based Relaxation of Continuous Disordered Systems
}

\author{
G. T. Barkema \\ Institute for Advanced Study, Olden Lane, Princeton, New Jersey 08540 \\ Normand Mousseau \\ Département de Physique et Groupe de Recherche en Physique et Technologie des Couches Minces (GCM), \\ Université de Montréal, Montréal, Québec, Canada, H3C 3J7
}

(Received 23 July 1996)

\begin{abstract}
A computational approach is presented to obtain energy-minimized structures in glassy materials. This approach, the activation-relaxation technique (ART), achieves its efficiency by focusing on significant changes in the microscopic structure (events). The application of ART is illustrated with two examples: the structure of amorphous silicon and the structure of $\mathrm{Ni}_{80} \mathrm{P}_{20}$, a metallic glass. [S0031-9007(96)01706-1]
\end{abstract}

PACS numbers: 61.43.Dq, 02.70.Rw, 73.61.Jc., 82.20.Wt

Amorphous and glassy materials, from silica glasses to polymers and proteins, are of considerable fundamental and technological interest. Progress in the understanding of these materials has been impaired, however, by the very slow dynamics and relaxation they display. This has been especially true for computational studies where machine limitations remain important. In the last years, significant advances have been made in techniques to study the dynamics of disordered systems in a discrete space, like spin-glasses and reaction-diffusion models. Improvements like the Swendsen and Wang cluster algorithm [1] and the event-based method of Voter [2] and Barkema et al. [3] have increased the accessible time scale for these problems by many orders of magnitude. In contrast, no such development has been seen for disordered systems like glassy materials, polymers, or proteins. In these systems, what constitutes a change in configuration - a move-is generally ill defined due to the continuous space to which the atoms have access: Coordination, state, and defects fluctuate widely and cannot be characterized uniquely. The generic approach for studying these continuous disordered systems is molecular dynamics (MD), which is not sensitive to these problems of definition. However, time steps in MD simulations are set by the phonon period of 50 to 100 femtoseconds, and therefore MD simulations generally cannot reach the relaxation time scale at which many interesting phenomena happen.

Rather than following the irrelevant details of the molecular motion as atoms vibrate back and forth about their average position, we propose in this Letter a method which focuses on significant changes in the microscopic structure (events), i.e., those which imply crossing the barriers that impair relaxation. For disordered systems, which show slow evolution, this event-based technique therefore allows the study of relaxation on the system's own time scale, not that of the phonons. Of course, once events are defined, it is possible to either relax the network statically to a global minimum or to follow its long-time dynamics.
The activation-relaxation technique (ART) allows the system to evolve following well-defined paths between local energy minima. Starting in a local minimum, it proceeds in two steps. (1) Activation: The configuration is moved to a local saddle point. (2) Relaxation: The system is pushed over the saddle point and relaxed to a new minimum. By restricting its search of paths to those going through saddle points, ART focuses on the miniscule part of the configuration space which is physically accessible and therefore results in an efficiency which cannot be achieved by more conventional Monte Carlo moves.

We first discuss the implementation of ART in more detail. We then illustrate the application of ART by considering two test cases for glassy materials: amorphous silicon and $\mathrm{Ni}_{80} \mathrm{P}_{20}$, a metallic glass. We conclude with a discussion of other applications for ART.

Algorithm. - The underlying concept of ART is that it is the material itself, as defined by its total energy surface, which should determine events, not an external rule. This idea is realized through the following steps: We start with a configuration relaxed in a local energy minimum using a force $\vec{F}$ derived from an appropriate interaction potential (empirical or $a b$ initio). At the start of the activation, a single atom is slightly displaced along a random direction to create a nonzero term in the force. The configuration is then moved to a nearby saddle point by iterative application of a redefined force:

$$
\vec{G}=\vec{F}-(1+\alpha)(\vec{F} \cdot \Delta \hat{X}) \Delta \hat{X} .
$$

Here $\vec{F}$ is the $3 N$-dimensional force vector from the interaction potential, $\hat{X}$ is a $3 N$-dimensional unit vector pointing from the last local minimum to the current position of the configuration, and $\alpha$ is a positive number. The new force $\vec{G}$ is thus opposite in sign to $\vec{F}$ in the direction parallel to $\hat{X}$, and equal to $\vec{F}$ in any direction perpendicular to $\hat{X}$. At each iteration, $\vec{G}$ is evaluated and the atoms are moved according to it, therefore forcing the system to follow a valley up-hill in the energy landscape 
towards a saddle point (see Fig. 1). At the saddle point, both $\vec{G}$ and $\vec{F}$ are zero and the iterative process stops: The activation is complete. We then proceed with the relaxation by moving the system along $\vec{F}$ into a new local minimum.

Equation (1) involves all atoms of the system; it is therefore the whole configuration which is taken through a saddle point to a new minimum, not a fixed or predefined set of atoms. An event can be characterized a posteriori by counting how many atoms have been displaced significantly (by, say, more than $0.01 \AA$ ). In this way we have found events involving from one to a few hundred atoms, indicating that a wide range of excitations can indeed be sampled.

In effect, ART limits the configuration space as seen by the system. For an $N$-particle system, this space is a $3 \mathrm{~N}$-dimensional continuum; however, most of it is energetically highly unfavorable and thus never visited if the system is at low temperature. In fact the configuration is almost always located in or very near a local energy minimum. Thus the system is effectively confined to "dots" in the $3 N$-dimensional configuration space, corresponding to the local energy minima. Given enough time, however, the configuration will hop from one such local minimum to another nearby. To take this hopping into account, we connect the isolated dots by paths going through local saddle points, which are the most likely, thus creating a whole network of minima. This reduction of a $3 N$-dimensional space to an effective configuration space consisting of the local energy minima and paths between them makes possible the efficient relaxation obtained with ART. This does not mean that we ignore the phonons altogether. They remain indirectly present in ART, as they provide the energy for going through the saddle points, i.e., jumping the barriers. This is sufficient since the relaxation and dynamics of glasses and amorphous materials are orders of magnitude slower than the typical phonon period or, in other words, the energy barri-

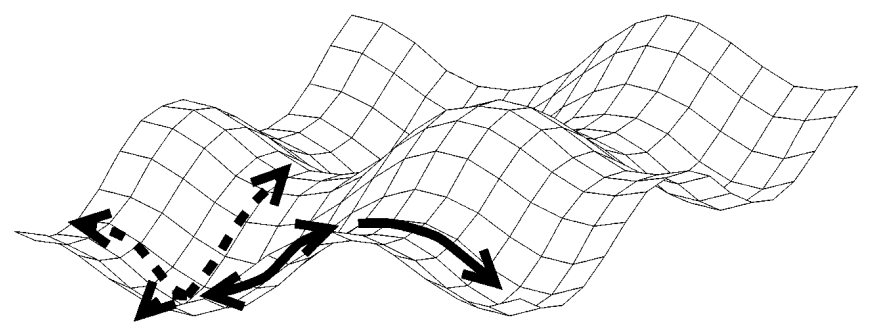

FIG. 1. Illustration of the activation and relaxation steps in ART for a two-dimensional energy landscape. The bottoms of the wells represent local minima. From each minimum, the system can reach four saddle points. The four arrows pointing up in the front right hand side indicate well the possible directions of $\vec{G}$ as defined in Eq. (1). Similarly, the downpointing arrows from the saddle point in the center indicate the directions of the force $\vec{F}$ toward a minimum energy. The fullline arrows show the path for a single event. ers in these systems are much larger than the temperature at which these materials are studied.

On a more technical side, we have chosen the Levenberg-Marquardt minimization technique both for activation to the saddle point and relaxation away from it. To perform global minimization, we used simulated annealing. The acceptance ratio is given by $P_{\text {accept }}=\min [1, \exp (-\Delta E / k T)]$, where $T$ is a fictive temperature. It is also necessary to avoid saddle points found in the very close vicinity of the minimum and which are an artifact of the static simulation. In an MD simulation these low saddle points would be washed out by the constant vibration of the lattice. In order to shun them, we introduced a repulsive harmonic potential which forces the system away from these unimportant saddle points, $E=A\left(|\Delta \vec{X}|-X_{c}\right)^{2}$ with a cutoff at $X_{c} .|\Delta \vec{X}|$ represents the displacement of the whole configuration, and $X_{c}$ is typically $0.5 \AA$.

Amorphous silicon. - Amorphous silicon $(a-\mathrm{Si})$ represents a good test-case for the application of ART to covalent amorphous materials. Many techniques have been used to study this material, including the static bond-switching algorithm of Wooten, Winer, and Weaire (WWW) [4], which proved very efficient in spite of the unphysical events it proposes and MD with a wide choice of interactions [5]. The MD models all suffer from the presence of a large number of defects, most of which, moreover, being floating bonds, i.e., overcoordinated atoms, which are not seen experimentally. This is particularly so when MD is carried out with empirical potentials. Even the best of those, developed by Stillinger and Weber (SW) [6], generally gives a structure with a coordination of 4.124.30. Tight-binding [7] and $a b$ initio MD [8] also show too many defects, but the coordination is normally lower; much longer relaxation with larger unit cells should result in a good agreement with experiment. The situation is not so clear in the case of empirical potentials, where doubts remain as to whether the problem comes from the potential itself or from too short simulations [9]. ART is well suited to address this question, since it does not suffer from the slow dynamics inherent to MD.

First, we show that ART can reproduce the structure found by MD using the SW potential. Starting from a 216-atom random closed packed configuration, with a density corresponding to the one of crystalline silicon (see top curve in Fig. 2), we applied ART until the system came to a stable energy. The radial distribution function (RDF) for this configuration is labeled $(B)$ in Fig. 2. The final energy, $-4.12 \mathrm{eV} /$ atom, is as low as what is obtained with MD [10], while the coordination of 4.17 is at the lower end of the spectrum. Although in good agreement with MD simulations, the overcoordination as well as the shoulder on the left side of the secondneighbor peak in the RDF (curve $B$ in Fig. 2) show that the configuration retains a strong liquidlike structure which is not present experimentally. 


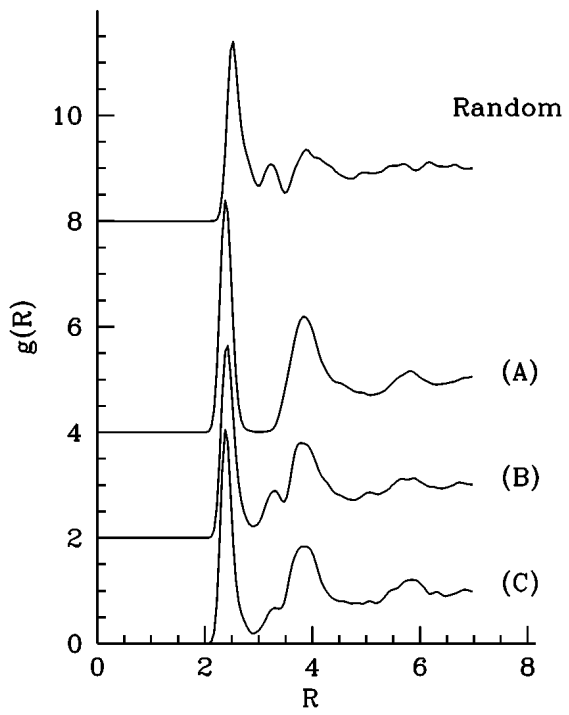

FIG. 2. Radial distribution function for a randomly packed system (top curve) and after three different energy minimizations: (a) after relaxation with a modified SW potential with ART, (b) after relaxation with the SW potential with ART, (c) relaxation with the $\mathrm{SW}$ potential with ART, starting from (a).

To show that this failure to reach the experimental structure is not due to ART itself, we performed a second simulation with an ad hoc modification to the SW potential: The three-body contribution to the total interaction energy was doubled. The effect of this modification is to increase the cost of overcoordination, helping the system to move away from the liquidlike phase. In order to test the scaling of the method with size, we applied ART, with this modified potential, to the 216-atom random closed packed configuration discussed in the previous paragraph and to a 1000-atom one. Both networks necessitated about 5 ART events per atom to reach a stable point showing that the activation-relaxation technique is not slowed down with increasing size. Further simulation did not change the properties of those networks, while structural properties for the two lattices were essentially the same. The RDF for the 1000-atom configuration is plotted as curve $(A)$ in Fig. 2. This quantity, as well as an almost perfect coordination of 3.97 and a rms angular deviation of 9.97 degrees, is in excellent agreement with experiment.

Further insight into the cause of the discrepancies between experiments and SW-based MD simulations was obtained by a third minimization. Starting this time from the 216-atom configuration obtained with modified SW, we relaxed it using the conventional SW parameters. The result, curve $C$ in Fig. 2, shows a liquidlike structure very similar to the one obtained in $(B)$ with an equivalent energy per atom of $-4.12 \mathrm{eV}$ : ART converged towards the same point starting from completely different initial configurations.

These results indicate clearly that ART can move through the configuration space towards a minimum in spite of large barriers. We could recover the characteristic structure for the SW from two completely different initial configurations. Moreover, with a modified potential, it was possible to obtain an amorphous structure in good agreement with experiment. As a result of our ART simulations we showed that the structural differences between MD with empirical potentials and experiments are caused, in part, by defective potentials, as was proposed in Ref. [9]. We expect that ART simulations with semi-empirical tight-binding interactions or $a b$ initio potentials will also produce a network with a structure close to that observed experimentally.

A final point about these simulations. Since the density of the final configuration does not have to be the same as for crystalline silicon ( $c$-Si), we also let the volume relax as one additional degree of freedom, i.e., the cell remains cubic but fluctuates in size. In the first simulation, with SW parameters, this freedom resulted in a density about 4.5 percent larger than that of $c$-Si, while the stronger three-body term in the second simulation leads to a density which is about five percent lower. The final density of configuration in the last simulation is essentially equal to the crystalline one. This supports the usual view that the energy landscape of disordered systems has multiple minima that are very close in energy.

Glassy $\mathrm{Ni}_{80} \mathrm{P}_{20}$. - As the second test-case of ART, we considered, a prototypical metallic glass which has been the subject of considerable investigation (e.g., [11,12]). The structure of this glass corresponds to the dense random packing model proposed by Bernal [13]. Its topology is controlled by local packing, not covalent bonding, and is thus relevant for high coordination glasses. The more compact environment leads to a topological rigidity larger than that for amorphous semiconductors [14], which makes barriers higher.

We selected a Lennard-Jones (L-J) potential with the parameters used by Kob and Andersen [12]. This allows for a direct comparison with their MD simulation that lasted $60 \mathrm{~ns}$. As in the previous section, we started from a purely random configuration of 250 atoms with the proper density and evolved it with ART using simulated annealing. The volume was allowed to adjust. The top four curves in Fig. 3 show the evolution of the RDF for the P-P distances during the minimization process. We can see that there is considerable change in the coordination of $\mathrm{P}$ atoms as the total configurational energy decreases.

As discussed recently by Angell [15], the lack of lowenergy disordered configurations hinders the study of rapid and slow dynamics in glasses far away from the glass temperature. The final ART configuration has an energy of $-7.681 \mathrm{~L}-\mathrm{J}$ units providing a significant improvement over the excellent MD simulation of Kob and Andersen, which reaches an energy of $-7.667 \mathrm{~L}-\mathrm{J}$ units when relaxed to zero temperature (see the bottom curve of Fig. 3). From Fig. 3, one can also see that the number of P-P first neighbors 


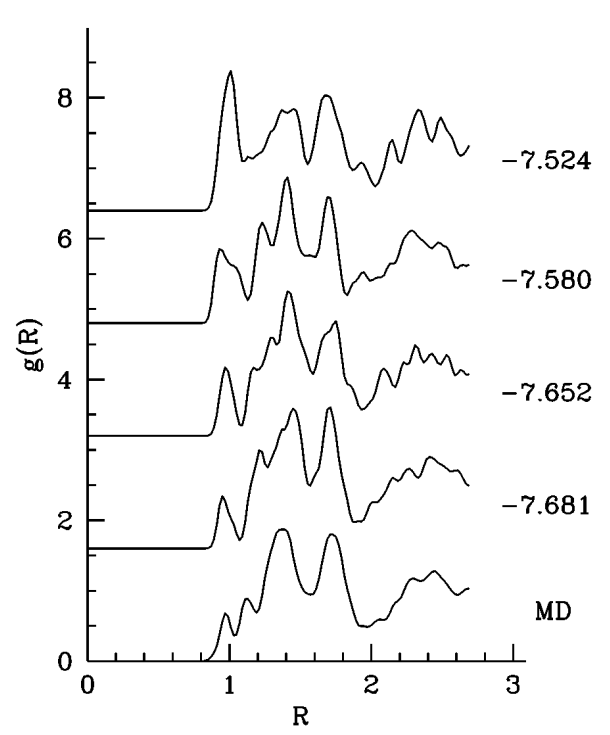

FIG. 3. Evolution of the radial distribution function of the P$\mathrm{P}$ distances during the ART simulation. The energy, shown to the right of each curve, is in Lennard-Jones units. The bottom curve is the RDF of a zero-temperature relaxed MD configuration of 1000 atoms, obtained from Kob and Andersen, with an energy of -7.667 L-J units.

decreases as these atoms diffuse away from each other in an important rearrangement of the lattice. ART can thus be used to obtain a low-energy structure which can then be studied dynamically either via molecular or event-based dynamics.

Conclusion. - We have presented a computational approach to the relaxation of continuous disordered systems that we call activation-relaxation technique (ART). ART is not based on any specific potential or structure and can therefore be applied to a wide range of systems. In two test-cases, the relaxation of $a-\mathrm{Si}$ and $\mathrm{Ni}_{80} \mathrm{P}_{20}$ starting from random packed configurations, ART has provided new insights. It resolved a long-standing question about the cause of the liquidlike nature of SW-based MD-relaxed $a$-Si. It also produced $\mathrm{Ni}_{80} \mathrm{P}_{20}$ samples with a significantly lower energy than MD, showing the way for creating weakly strained configurations for disordered structures.

For which systems would ART be appropriate? The natural time scale of ART is event-based, that of MD is phonon-based. This makes the two techniques complementary: For a system with short-time dynamics, MD is preferable; when rare events dominate, ART has to be applied.

In the near future we will combine ART with tightbinding calculations for the study of $a-\mathrm{Si}$. This will be extended to other disordered materials like $a$-GaAs and $\mathrm{SiO}_{2}$. Future work will include the relaxation of polymers and proteins inside and outside solution. We also want to combine ART with rare-event dynamics [3] so that dynamical quantities like self-diffusion can be obtained with the efficiency of ART.

We thank G. Boisvert, J.L. Brebner, and L. J. Lewis for critical reading of this manuscript, and W. Kob for sending us his $\mathrm{Ni}_{80} \mathrm{P}_{20}$ configuration. G. T.B. thanks T. Arias for useful discussions and acknowledges support from the DOE under Grant No. DE-FG02-90ER40542 and from the Monell foundation.

[1] R. H. Swendsen and J.-S. Wang, Phys. Rev. Lett. 58, 86 (1987).

[2] A. F. Voter, Phys. Rev. B 34, 6819 (1986).

[3] G. T. Barkema, M. J. Howard, and J. L. Cardy, Phys. Rev. E 53, 2017 (1996).

[4] F. Wooten, K. Winer, and D. Weaire, Phys. Rev. Lett. 54, 1392 (1985).

[5] R. Biswas, G. S. Grest, and C. M. Soukoulis, Phys. Rev. B 36, 7437 (1987); W. D. Luetdke and U. Landman, Phys. Rev. B 37, 4656 (1988); S. J. Cook and P. Clancy, Phys. Rev. B 47, 7686 (1993).

[6] F. H. Stillinger and T. A. Weber, Phys. Rev. B 31, 5262 (1985).

[7] J. M. Mercer, Jr. and M. Y. Chou, Phys. Rev. B 43, 6768 (1991); G. Servalli and L. Colombo, Europhys. Lett. 22, 107 (1993); E. Kim and Y.H. Lee, Phys. Rev. B 49, 1743 (1994).

[8] R. Car and M. Parrinello, Phys. Rev. Lett. 60, 204 (1988); P. A. Fedders, D. A. Drabold, O. F. Sankey, and J. D. Dow, Phys. Rev. B 42, 5135 (1990).

[9] W. D. Luedtke and U. Landman, Phys. Rev. B 40, 1164 (1989).

[10] M. D. Kluge, J. R. Ray, and A. Rahman, Phys. Rev. B 36, 4234 (1987).

[11] L. J. Lewis, Phys. Rev. B 44, 4245 (1991).

[12] W. Kob and H.C. Andersen, Phys. Rev. E 51, 4626 (1995).

[13] J. D. Bernal, Nature (London) 188, 910 (1960).

[14] N. Mousseau and M.F. Thorpe, Phys. Rev. B 45, 2015 (1992).

[15] C. A. Angell, Comput. Mater. Sci. 4, 285 (1995). 